\title{
Entropy Generation and Mathematical Inequalities
}

\author{
Xiaowei Tian ${ }^{1,2}$, Liqiu Wang ${ }^{1,2}$ \\ ${ }^{1}$ Department of Mechanical Engineering, the University of Hong Kong \\ Hong Kong \\ txwsky@hku.hk; lqwang@hku.hk \\ ${ }^{2} \mathrm{HKU}-$ Zhejiang Institute of Research and Innovation (HKU-ZIRI) \\ Hangzhou, China
}

\begin{abstract}
In this paper, we examine the entropy generation regarding its magnitude and the limit as time tends to infinity and apply the second law of thermodynamics to develop mathematical inequalities with heat conduction in adiabatic spheres. The former shows a bounded entropy generation if the heat conduction is initiated by the initial temperature distribution, but unbounded if the heat conduction involves a heat source with positive volume average over the sphere. The latter yields various innovative relations that are useful both for studying differential equations and for examining accuracy of analytical, numerical and experimental results. The work not only builds up the relation between the second law of thermodynamics and mathematical inequalities, but also offers some fundamental insights of universe and our future.
\end{abstract}

Keywords: Entropy Generation, Heat Conduction, Mathematical Inequalities, Second Law of Thermodynamics.

\section{Introduction}

As an important transport process, heat conduction is governed by the first and the second laws of thermodynamics [13]. With the classical Fourier's law of heat conduction as the constitutive relation of heat flux density, the relation between the heat flux density vector and the temperature gradient $[1,4]$, the first law of thermodynamics yields the classical heatconduction equation whose solution provides the temperature field $[1,3]$. The application of the Fourier's law of heat conduction will then lead to heat transfer rate and the way to control it $[1,3]$.

Applied to heat conduction, the second law of thermodynamics states that: the entropy generation $S_{\text {gen }}$ of a system during heat conduction always increases, or, in the limiting case of a reversible process, remains constant, i.e., $d S_{\text {gen }} / d t \geq$ 0 with $t$ being the time [5,6]. This requires that $S_{\text {gen }}\left(t_{2}\right) \geq S_{\text {gen }}\left(t_{1}\right)$ for all $t_{2} \geq t_{1}$. With knowing temperature field from the heat-conduction equation, the entropy generation $S_{\text {gen }}$ becomes available [5,6]. Applying $d S_{\text {gen }} / d t \geq 0$ and $S_{\text {gen }}\left(t_{2}\right) \geq S_{\text {gen }}\left(t_{1}\right)$ can then yield mathematical inequalities and thus solution features of heat-conduction equations [2].

Energy is conserved by the first law of thermodynamics $[1,5,6]$. The very essence of entropy is the part of system energy that cannot be transformed into useful work [7]. Any entropy generation will then degrade the quality of energy. It becomes thus significant and relevant to examine $d S_{\text {gen }} / d t$ regarding the way to reduce its magnitude and $\lim _{t \rightarrow \infty} S_{\text {gen }}$ regarding whether it is bounded or not.

The present work aims to develop above-mentioned mathematical inequalities and examine $d S_{\text {gen }} / d t$ and $\lim _{t \rightarrow \infty} S_{\text {gen }}$ with heat conduction in three-dimensional sphere. Note that such an analysis is very limited in the literature and differs fundamentally from other studies of the second law analysis that mainly aim for improving performance of practical processes [8-18]. In Section 2, we make analytical derivation of temperature field, entropy generation and its limit, and mathematical inequalities. Our derivation is made for the heat conduction driven by the initial temperature distribution, by the internal source and by the both, respectively, with the more details being given for the first case. In section 3, we summarize the inequalities developed in Section 2 and the physical implication of the entropy generation and its limit obtained in Section 2. We draw some concluding remarks in Section 4. 


\section{Temperature Field, Entropy Generation and Mathematical Inequalities}

Consider one dimensional heat conduction along the radial direction in a sphere of radius a with constant material properties and specified temperature gradient at the boundary, the second kind or Neumann boundary condition [1]. As the contribution of nonhomogeneous boundary condition to the temperature field can be represented by source and initial terms [2], we can focus our attention to the following initial-boundary value problem with homogeneous boundary conditions in spherical coordinates, shown in Fig. 1, without loss of the generality:

$$
\left\{\begin{array}{l}
T_{\mathrm{t}}=a_{0}{ }^{2} \Delta T+f(r, t), \quad(0, a) \times(0,+\infty) \\
\left.T_{r}\right|_{r=a}=0 \\
\left.T\right|_{t=0}=\varphi(r) .
\end{array}\right.
$$

where $\mathrm{t}$ and $\mathrm{T}$ are time and temperature, respectively. $a_{0}^{2}$ is the thermal diffusivity. $\varphi(r)$ is the initial temperature distribution over the sphere. $f(r, t)$ is the rate of heat generation inside the sphere per unit volume and per unit specific capacity of the material. The heat generation may be due to nuclear, electrical, chemical, gammy-ray, or other sources that may be a function of time and/or position.
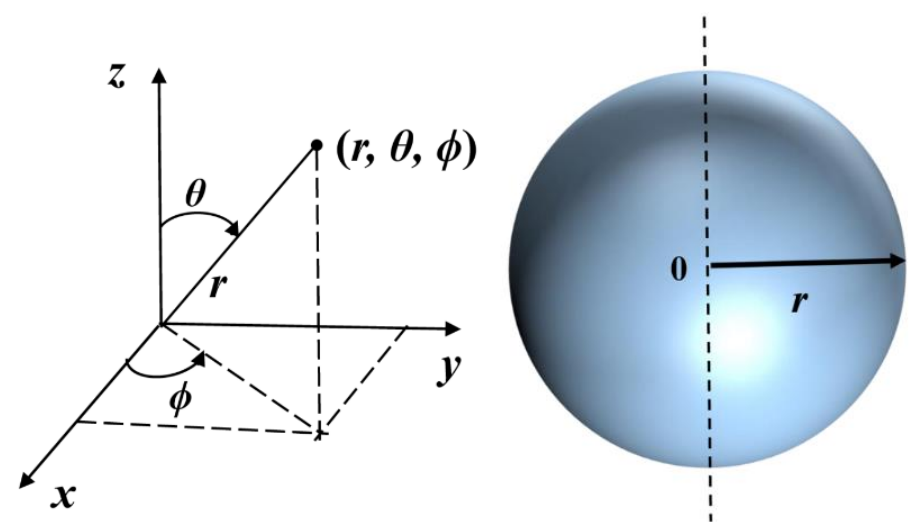

Fig. 1: Heat conduction in adiabatic spheres along the r-direction $(\theta=\pi, \phi=2)$ and spherical coordinate system.

\subsection{Heat Conduction Initiated by the Initial Temperature Distribution}

For the heat conduction driven by the initial temperature distribution, $f(r, t)=0$, and Eq. (1) reduces into

$$
\left\{\begin{array}{l}
T_{\mathrm{t}}=a_{0}^{2} \Delta T \\
\left.T_{r}\right|_{r=a}=0 \\
\left.T\right|_{t=0}=\varphi(r)
\end{array}\right.
$$

To obtain the solution of (2), consider $T(r, t)=T(t) R(r)$, applying the superposition principle and initial condition with the completeness and the orthogonality of the eigenfunction group and its normal square yield the solution of (2),

$$
\left\{\begin{array}{l}
T_{\varphi}(r, t)=\sum_{l=0}^{\infty} A_{l} e^{-\omega_{l}^{2} t} j_{0}\left(k_{l} r\right) \\
A_{l}=\frac{1}{M_{l}} \int_{0}^{a} \varphi(r) j_{0}\left(k_{l} r\right) r^{2} d r
\end{array}\right.
$$

where $k_{l}=\mu_{l}^{(1 / 2)} / a, \omega_{l}=k_{l} a_{0}, j_{0}\left(k_{l} r\right)$ is spherical Bessel equation of order 0 and $M_{l}$ is the normal square of $j_{0}\left(k_{l} r\right)$. 


$$
\left\{\begin{array}{l}
M_{l}=\frac{\pi a^{3}}{4 \mu_{l}} J_{\frac{1}{2}}^{2}\left(\mu_{l}\right), \quad l=1,2,3, \ldots \\
M_{0}=\frac{a^{3}}{3}, \quad l=0
\end{array}\right.
$$

Entropy analysis and mathematical inequalities

The total entropy in the sphere is $[5,6]$,

$$
S_{\varphi}(t)=4 \pi \rho C_{V} \int_{0}^{a}\left[\ln T_{\varphi}(r, t)\right] r^{2} d r=4 \pi \rho C_{V} \int_{0}^{a}\left[\ln \sum_{l=0}^{\infty} A_{l} e^{-\omega_{l}^{2} t} j_{0}\left(k_{l} r\right)\right] r^{2} d r
$$

Note that $S_{\varphi}(t)$ is also the total entropy generation for the case of adiabatic boundary conditions with vanished thermal entropy flux [5, 6]. Applying Eq. (3), we obtain

$$
\lim _{t \rightarrow+\infty} S_{\varphi}(t)=4 \pi \rho C_{V} \int_{0}^{a}\left[\ln \lim _{t \rightarrow+\infty} T_{\varphi}(r, t)\right] r^{2} d r=4 \pi \rho C_{V} \int_{0}^{a} \ln A_{0} r^{2} d r
$$

When $l=0$,

$$
M_{l}=\frac{a^{3}}{3}, j_{0}(0)=1
$$

Therefore,

$$
\mathrm{A}_{0}=\frac{1}{\frac{a^{3}}{3}} \int_{0}^{a} \varphi(r) j_{0}(0) r^{2} d r=\frac{3}{a^{3}} \int_{0}^{a} \varphi(r) r^{2} d r
$$

which is the volume average of the initial temperature distribution $\varphi(r)$ over the sphere, and

$$
\lim _{t \rightarrow+\infty} S_{\varphi}(t)=4 \pi \rho C_{V} \int_{0}^{a}\left[\ln \frac{3}{a^{3}} \int_{0}^{a} \varphi(r) r^{2} d r\right] r^{2} d r=\frac{4 \pi a^{3}}{3} \rho C_{V} \ln \frac{3}{a^{3}} \int_{0}^{a} \varphi(r) r^{2} d r
$$

which is bounded and equal to system total entropy at a uniform average temperature of the initial temperature distribution over the whole sphere.

Note that,

$$
\frac{d S_{\varphi}(t)}{d t}=4 \pi \rho C_{V} \int_{0}^{a} \frac{1}{T_{\varphi}(r, t)} \frac{\partial T_{\varphi}(r, t)}{\partial t} r^{2} d r=4 \pi \rho C_{V} \int_{0}^{a} \frac{\sum_{l=0}^{\infty} A_{l}\left(-\omega_{l}^{2}\right) e^{-\omega_{l}^{2} t} j_{0}\left(k_{l} r\right)}{\sum_{l=0}^{\infty} A_{l} e^{-\omega_{l}^{2} t} j_{0}\left(k_{l} r\right)} r^{2} d r
$$

Applying the principle of entropy increase $d S_{\varphi}(t) / d t \geq 0$ leads to,

$$
\int_{0}^{a} \frac{\sum_{l=0}^{\infty} A_{l}\left(-\omega_{l}^{2}\right) e^{-\omega_{l}^{2} t} j_{0}\left(k_{l} r\right)}{\sum_{l=0}^{\infty} A_{l} e^{-\omega_{l}^{2} t} j_{0}\left(k_{l} r\right)} r^{2} d r \geq 0
$$

As $d S_{\varphi}(t) / d t \geq 0$, we also have 


$$
S_{\varphi}\left(t_{1}\right) \leq S_{\varphi}\left(t_{2}\right) \quad \forall 0<t_{1} \leq t_{2}<+\infty
$$

This yields the

$$
\int_{0}^{a}\left[\ln \sum_{l=0}^{\infty} A_{l} e^{-\omega_{l}^{2} t_{1}} j_{0}\left(k_{l} r\right)\right] r^{2} d r \leq \int_{0}^{a}\left[\ln \sum_{l=0}^{\infty} A_{l} e^{-\omega_{l}^{2} t_{2}} j_{0}\left(k_{l} r\right)\right] r^{2} d r
$$

Note that

$$
S_{\varphi}(0)=4 \pi \rho C_{V} \int_{0}^{a}\left[\ln T_{\varphi}(r, 0)\right] r^{2} d r=4 \pi \rho C_{V} \int_{0}^{a}[\ln \varphi(r)] r^{2} d r, \quad S_{\varphi}(+\infty)=\frac{4 \pi a^{3}}{3} \rho C_{V} \ln \frac{3}{a^{3}} \int_{0}^{a} \varphi(r) r^{2} d r
$$

Applying $S_{\varphi}(0) \leq S_{\varphi}(+\infty)$ leads to

$$
4 \pi \rho C_{V} \int_{0}^{a}[\ln \varphi(r)] r^{2} d r \leq \frac{4 \pi a^{3}}{3} \rho C_{V} \ln \frac{3}{a^{3}} \int_{0}^{a} \varphi(r) r^{2} d r
$$

that is

$$
\frac{3}{a^{3}} \int_{0}^{a}[\ln \varphi(r)] r^{2} d r \leq \ln \frac{3}{a^{3}} \int_{0}^{a} \varphi(r) r^{2} d r
$$

Eq.(16) is the one-dimensional extension over the sphere of well-known Arithmetic-mean---geometric-mean inequality $[19,20]$ and can be proven mathematically as following as well.

\subsection{Heat Conduction Driven by the Internal Source}

For the heat conduction driven exclusively by the internal source, $\varphi(r)=0$ and Eq. (1) reduces

$$
\left\{\begin{array}{l}
T_{\mathrm{t}}=a_{0}^{2} \Delta T+f(r, t) \\
\left.T_{r}\right|_{r=a}=0 \\
\left.T\right|_{t=0}=0
\end{array}\right.
$$

By the property of $\delta$ function and superposition principle, Eq. (15) thus has the solution of

$$
\left\{\begin{array}{l}
T_{f}(r, t)=\int_{0}^{t} \sum_{l=0}^{\infty} B_{l} e^{-\omega_{l}^{2}(t-\tau)} j_{0}\left(k_{l} r\right) d \tau \\
B_{n l}=\frac{1}{M_{l}} \int_{0}^{a} f(r, \tau) j_{0}\left(k_{l} r\right) r^{2} d r
\end{array}\right.
$$

where $T_{f}$ stands for the temperature for heat conduction driven exclusively by the internal heat source, $k_{l}=\mu_{l}^{(1 / 2)} / a, \omega_{l}=$ $k_{l} a_{0}$.

\section{Entropy analysis and mathematical inequalities}

The total entropy in the sphere at time instant $t$ is, which is also the entropy generation up to time instant $t$, 


$$
S_{f}(t)=4 \pi \rho C_{V} \int_{0}^{a}\left[\ln T_{f}(r, t)\right] r^{2} d r=S_{f}(t)=4 \pi \rho C_{V} \int_{0}^{a}\left[\ln \int_{0}^{t} \sum_{l=0}^{\infty} B_{l} e^{-\omega_{l}^{2}(t-\tau)} j_{0}\left(k_{l} r\right) d \tau\right] r^{2} d r
$$

By applying Eq.(19)

$$
\begin{gathered}
\lim _{t \rightarrow+\infty} S_{f}(t)=4 \pi \rho C_{V} \int_{0}^{a}\left[\ln \lim _{t \rightarrow+\infty}\left(\int_{0}^{t} \sum_{l=0}^{\infty} B_{l} e^{-\omega_{l}^{2}(t-\tau)} j_{0}\left(k_{l} r\right) d \tau\right)\right] r^{2} d r \\
=4 \pi \rho C_{V} \int_{0}^{a}\left[\ln \lim _{t \rightarrow+\infty}\left(\int_{0}^{t} \frac{3}{a^{3}} \int_{0}^{a} f(r, \tau) r^{2} d r d \tau\right)\right] r^{2} d r
\end{gathered}
$$

Also,

$$
\frac{d S_{f}(t)}{d t}=4 \pi \rho C_{V} \int_{0}^{a} \frac{1}{T_{f}(r, t)} \frac{\partial T_{f}(r, t)}{\partial t} r^{2} d r=4 \pi \rho C_{V} \int_{0}^{a} \frac{\int_{0}^{t} \sum_{l=0}^{\infty} B_{l}\left(-\omega_{l}^{2}\right) e^{-\omega_{l}^{2}(t-\tau)} j_{0}\left(k_{l} r\right) d \tau+f(r, t)}{\int_{0}^{t} \sum_{l=0}^{\infty} B_{l} e^{-\omega_{l}^{2}(t-\tau)} j_{0}\left(k_{l} r\right) d \tau} r^{2} d r
$$

By the second law of thermodynamics, $d S_{f}(t) / d t \geq 0$. We have thus, by noting that $\rho C_{V}>0$,

$$
\int_{0}^{a} \frac{\int_{0}^{t} \sum_{l=0}^{\infty} B_{l}\left(-\omega_{l}^{2}\right) e^{-\omega_{l}^{2}(t-\tau)} j_{0}\left(k_{l} r\right) d \tau+f(r, t)}{\int_{0}^{t} \sum_{l=0}^{\infty} B_{l} e^{-\omega_{l}^{2}(t-\tau)} j_{0}\left(k_{l} r\right) d \tau} r^{2} d r \geq 0
$$

As $d S_{f} / d t \geq 0$, we also have

$$
S_{f}\left(t_{1}\right) \leq S_{f}\left(t_{2}\right) \quad \forall 0<t_{1} \leq t_{2}<+\infty
$$

We thus obtain the following inequality:

$$
\int_{0}^{a}\left[\ln \left(\int_{0}^{t_{1}} \sum_{l=0}^{\infty} B_{l} e^{-\omega_{l}^{2}\left(t_{1}-\tau\right)} j_{0}\left(k_{l} r\right) d \tau\right)\right] r^{2} d r \leq \int_{0}^{a}\left[\ln \left(\int_{0}^{t_{2}} \sum_{l=0}^{\infty} B_{l} e^{-\omega_{l}^{2}\left(t_{2}-\tau\right)} j_{0}\left(k_{l} r\right) d \tau\right)\right] r^{2} d r
$$

Also,

$$
\begin{gathered}
S_{f}(0)=4 \pi \rho C_{V} \int_{0}^{a}\left[\ln \lim _{t \rightarrow+0} T_{f}(r, t)\right] r^{2} d r=-\infty \\
S_{f}(+\infty)=4 \pi \rho C_{V} \int_{0}^{a}\left[\ln \lim _{t \rightarrow+\infty}\left(\int_{0}^{t} \frac{3}{a^{3}} \int_{0}^{a} f(r, \tau) r^{2} d r d \tau\right)\right] r^{2} d r
\end{gathered}
$$

As $S_{f}(0) \leq S_{f}(+\infty)$, we arrive at:

$$
4 \pi \rho C_{V} \int_{0}^{a}\left[\ln \lim _{t \rightarrow+\infty}\left(\int_{0}^{t} \frac{3}{a^{3}} \int_{0}^{a} f(r, \tau) r^{2} d r d \tau\right)\right] r^{2} d r \geq-\infty
$$


Let's now examine $S_{f}(+\infty)$ in details. For sufficiently large $t$,

$$
S_{f}(t)=4 \pi \rho C_{V} \int_{0}^{a}\left[\ln \left(\int_{0}^{t} \frac{3}{a^{3}} \int_{0}^{a} f(r, \tau) r^{2} d r d \tau\right)\right] r^{2} d r=\frac{4 \pi a^{3}}{3} \rho C_{V} \ln \left(\int_{0}^{t} \frac{3}{a^{3}} \int_{0}^{a} f(r, \tau) r^{2} d r d \tau\right)
$$

Consider now the source with a positive volume average over the sphere so that for any time instant $\tau$, there exists a positive value $\varepsilon$ such that

$$
\frac{3}{a^{3}} \int_{0}^{a} f(r, \tau) r^{2} d r \geq \varepsilon>0 \text { or } \frac{3}{a^{3}} \int_{0}^{t} d \tau \int_{0}^{a} f(r, \tau) r^{2} d r \geq \varepsilon>0 \geq \varepsilon t
$$

Thus we have

$$
S_{f}(t) \geq \frac{4 \pi a^{3}}{3} \rho C_{V} \ln (\varepsilon t)
$$

As $\varepsilon>0$, عt can be sufficiently large for sufficiently large $t$. Note also that $\ln x$ increases always with $x$. We conclude, with Eq. (30),

$$
\forall M>0, \exists t_{0} \text {, when } t_{0}<t, M<S_{f}(t)
$$

that is

$$
\lim _{t \rightarrow+\infty} S_{f}(t)=S_{f}(+\infty)=+\infty
$$

\subsection{Heat Conduction Driven by the Initial Temperature Distribution and Internal Source}

The temperature field subjected to the effect of both initial temperature distribution and the source term is, by the superimposition principle,

$$
T_{\varphi f}(r, t)=T_{\varphi}(r, t)+T_{f}(r, t)
$$

With Eqs. (3) and (18), we have

$$
T_{\varphi f}(r, \theta, t)=\sum_{l=0}^{\infty} A_{l} e^{-\omega_{l}^{2} t} j_{0}\left(k_{l} r\right)+\int_{0}^{t} \sum_{l=0}^{\infty} B_{l} e^{-\omega_{l}^{2}(t-\tau)} j_{0}\left(k_{l} r\right) d \tau
$$

where $k_{l}=\mu_{l}^{\left(\frac{1}{2}\right)} / a, \omega_{l}=k_{l} a_{0}$

Entropy analysis and mathematical inequalities

The total entropy in the sphere at time instant $t$ is, which is also the entropy generation up to time instant $t$,

$$
S_{\varphi f}(t)=4 \pi \rho C_{V} \int_{0}^{a}\left[\ln \left(\sum_{l=0}^{\infty} A_{l} e^{-\omega_{l}^{2} t} j_{0}\left(k_{l} r\right)+\int_{0}^{t} \sum_{l=0}^{\infty} B_{l} e^{-\omega_{l}^{2}(t-\tau)} j_{0}\left(k_{l} r\right) d \tau\right)\right] r^{2} d r .
$$

Also, 


$$
\lim _{t \rightarrow+\infty} S_{\varphi f}(t)=4 \pi \rho C_{V} \int_{0}^{a}\left[\ln \lim _{t \rightarrow+\infty} T_{\varphi f}(r, t)\right] r^{2} d r=4 \pi \rho C_{V} \int_{0}^{a}\left[\ln \left(A_{0}+\lim _{t \rightarrow+\infty} \int_{0}^{t} B_{0} d \tau\right)\right] r^{2} d r
$$

where

$$
A_{0}=\frac{3}{a^{3}} \int_{0}^{a}[\varphi(r, \theta)] r^{2} d r, \quad B_{0}=\frac{3}{a^{3}} \int_{0}^{a}[f(r, \tau)] r^{2} d r
$$

Note that

$$
\begin{aligned}
& \frac{d S_{\varphi f}(t)}{d t}=4 \pi \rho C_{V} \int_{0}^{a}\left[\frac{1}{T_{\varphi f}(r, t)}\left(\frac{\partial T_{\varphi f}(r, t)}{\partial t}\right)\right] r^{2} d r \\
= & 4 \pi \rho C_{V} \int_{0}^{a}\left[\frac{\sum C_{l}\left(-\omega_{l}^{2}\right) e^{-\omega_{l}^{2} t}+\int_{0}^{t} \sum D_{l}\left(-\omega_{l}^{2}\right) e^{-\omega_{l}^{2}(t-\tau)} d \tau+f(r, t)}{\sum C_{l} e^{-\omega_{l}^{2} t}+\int_{0}^{t} \sum D_{l} e^{-\omega_{l}^{2}(t-\tau)} d \tau}\right] r^{2} d r
\end{aligned}
$$

where

$$
\sum C_{l}=\sum_{l=0}^{\infty} A_{l} j_{0}\left(k_{l} r\right) ; \sum D_{l}=\sum_{l=0}^{\infty} B_{l} j_{0}\left(k_{l} r\right)
$$

By the second law of thermodynamics that requires $d S_{\varphi f}(t) / d t \geq 0$, we have

$$
\int_{0}^{a}\left[\frac{\sum C_{l}\left(-\omega_{l}^{2}\right) e^{-\omega_{l}^{2} t}+\int_{0}^{t} \sum D_{l}\left(-\omega_{l}^{2}\right) e^{-\omega_{l}^{2}(t-\tau)} d \tau+f(r, t)}{\sum C_{l} e^{-\omega_{l}^{2} t}+\int_{0}^{t} \sum D_{l} e^{-\omega_{l}^{2}(t-\tau)} d \tau}\right] r^{2} d r \geq 0
$$

By applying

$$
S_{\varphi f}\left(t_{1}\right) \leq S_{\varphi f}\left(t_{2}\right), \quad \forall 0<t_{1} \leq t_{2}<+\infty
$$

we obtain

When $t_{1}=0, t_{2}=+\infty$,

$$
\begin{aligned}
& \int_{0}^{a}\left[\ln \left(\sum_{l=0}^{\infty} A_{l} e^{-\omega_{l}^{2} t_{1}} j_{0}\left(k_{l} r\right)+\int_{0}^{t} \sum_{l=0}^{\infty} B_{l} e^{-\omega_{l}^{2}\left(t_{1}-\tau\right)} j_{0}\left(k_{l} r\right) d \tau\right)\right] r^{2} d r \\
& \leq \int_{0}^{a}\left[\ln \left(\sum_{l=0}^{\infty} A_{l} e^{-\omega_{l}^{2} t_{2}} j_{0}\left(k_{l} r\right)+\int_{0}^{t} \sum_{l=0}^{\infty} B_{l} e^{-\omega_{l}^{2}\left(t_{2}-\tau\right)} j_{0}\left(k_{l} r\right) d \tau\right)\right] r^{2} d r
\end{aligned}
$$

$$
\begin{gathered}
S_{\varphi f}(0)=4 \pi \rho C_{V} \int_{0}^{a}\left[\ln T_{\varphi f}(r, 0)\right] r^{2} d r=4 \pi \rho C_{V} \int_{0}^{a}[\ln \varphi(r)] r^{2} d r \\
S_{\varphi f}(+\infty)=4 \pi \rho C_{V} \int_{0}^{a}\left[\ln \left(A_{0}+\lim _{t \rightarrow+\infty} \int_{0}^{t} B_{0} d \tau\right)\right] r^{2} d r
\end{gathered}
$$

By applying $S_{\varphi f}(0) \leq S_{\varphi f}(+\infty)$, we thus have 


$$
\int_{0}^{a}[\ln \varphi(r)] r^{2} d r \leq \int_{0}^{a}\left[\ln \left(A_{0}+\lim _{t \rightarrow+\infty} \int_{0}^{t} B_{0} d \tau\right)\right] r^{2} d r
$$

Consider now the source with a positive volume average over the sphere so that for any time instant $\tau$, there exists a positive value $\varepsilon$ such that

$$
\frac{3}{a^{3}} \int_{0}^{a}[f(r, \tau)] r^{2} d r \geq \varepsilon>0
$$

By using the results in 2.1 and 2.2, we have, for sufficiently large $t$,

$$
S_{\varphi f}(t)=4 \pi \rho C_{V} \int_{0}^{a}\left[\ln \left(A_{0}+\int_{0}^{t} \frac{3}{a^{3}} \int_{0}^{a}[f(r, \tau)] r^{2} d r d \tau\right)\right] r^{2} d r \geq 4 \pi \rho C_{V} \int_{0}^{a}\left[\ln \left(A_{0}+\varepsilon t\right)\right] r^{2} d r
$$

As $\varepsilon>0$, عt can be sufficiently large for sufficiently large $t$. Note also that $\ln x$ increases always with $x$. Eq. (47) thus leads to

$$
\forall M>0, \exists t_{0}, \text { when } t_{0}<t, M<S_{\varphi f}(t)
$$

that is

$$
\lim _{t \rightarrow+\infty} S_{\varphi f}(t)=S_{f}(+\infty)=+\infty
$$

\section{Discussion}

Table 1 summarizes the mathematical inequalities obtained from the second law of thermodynamics with the heat conduction in a sphere driven by the initial temperature distribution, the internal source and the both, respectively. They are developed by applying the positive semi-definiteness of temporal derivative of entropy generation, the larger entropy generation at time instant $t_{2}$ than at $t_{1}$ for all $t_{2} \geq t_{1}$, and the large entropy generation at time infinity than at initial time instant of heat conduction as the statements of the second law of thermodynamics convenient to apply. We have also proven one of these inequalities [Eq. (16)] mathematically. They form a group of tools that could be useful for examining heat conduction equations and their solutions and serve as the benchmarks of confirming solutions from analytical, numerical and experimental approaches.

Table 1: Integral inequalities developed in adiabatic spheres.

\begin{tabular}{|c|c|c|}
\hline $\begin{array}{c}\text { Heat } \\
\text { conduction }\end{array}$ & $\begin{array}{c}\mathbf{2}^{\text {nd }} \text { law of } \\
\text { thermodynamics }\end{array}$ & Inequalities \\
\hline \multirow{2}{*}{$\begin{array}{c}\text { By initial } \\
\text { temperature } \\
\text { distribution }\end{array}$} & $\frac{d S_{\varphi}(t)}{d t} \geq 0$ & $\int_{0}^{a} \frac{\sum_{l=0}^{\infty} A_{l}\left(-\omega_{l}^{2}\right) e^{-\omega_{l}^{2} t} j_{0}\left(k_{l} r\right)}{\sum_{l=0}^{\infty} A_{l} e^{-\omega_{l}^{2} t} j_{0}\left(k_{l} r\right)} r^{2} d r \geq 0$ \\
\cline { 2 - 3 } & $S_{\varphi}\left(t_{1}\right) \leq S_{\varphi}\left(t_{2}\right)$ & $\int_{0}^{a}\left[\ln \sum_{l=0}^{\infty} A_{l} e^{-\omega_{l}^{2} t_{1}} j_{0}\left(k_{l} r\right)\right] r^{2} d r \leq \int_{0}^{a}\left[\ln \sum_{l=0}^{\infty} A_{l} e^{\left.-\omega_{l}^{2} t_{2} j_{0}\left(k_{l} r\right)\right] r^{2} d r}\right.$ \\
\cline { 2 - 3 } & $S_{\varphi}(0) \leq S_{\varphi}(+\infty)$ & $\frac{3}{a^{3}} \int_{0}^{a}[\ln \varphi(r)] r^{2} d r \leq \ln \frac{3}{a^{3}} \int_{0}^{a} \varphi(r) r^{2} d r$ \\
\hline
\end{tabular}




\begin{tabular}{|c|c|c|}
\hline \multirow{3}{*}{$\begin{array}{l}\text { By internal } \\
\text { source }\end{array}$} & $\frac{d S_{f}(t)}{d t} \geq 0$ & $\int_{0}^{a} \frac{\int_{0}^{t} \sum_{l=0}^{\infty} B_{l}\left(-\omega_{l}^{2}\right) e^{-\omega_{l}^{2}(t-\tau)} j_{0}\left(k_{l} r\right) d \tau+f(r, t)}{\int_{0}^{t} \sum_{l=0}^{\infty} B_{l} e^{-\omega_{l}^{2}(t-\tau)} j_{0}\left(k_{l} r\right) d \tau} r^{2} d r \geq 0$ \\
\hline & $S_{f}\left(t_{1}\right) \leq S_{f}\left(t_{2}\right)$ & $\begin{aligned} & \int_{0}^{a}\left[\ln \left(\int_{0}^{t_{1}} \sum_{l=0}^{\infty} B_{l} e^{-\omega_{l}^{2}\left(t_{1}-\tau\right)} j_{0}\left(k_{l} r\right) d \tau\right)\right] r^{2} d r \\
\leq & \int_{0}^{a}\left[\ln \left(\int_{0}^{t_{2}} \sum_{l=0}^{\infty} B_{l} e^{-\omega_{l}^{2}\left(t_{2}-\tau\right)} j_{0}\left(k_{l} r\right) d \tau\right)\right] r^{2} d r\end{aligned}$ \\
\hline & $S_{f}(0) \leq S_{f}(\infty)$ & $4 \pi \rho C_{V} \int_{0}^{a}\left[\ln \lim _{t \rightarrow+\infty}\left(\int_{0}^{t} \frac{3}{a^{3}} \int_{0}^{a} f(r, \tau) r^{2} d r d \tau\right)\right] r^{2} d r \geq-\infty$ \\
\hline \multirow{3}{*}{$\begin{array}{l}\text { By both initial } \\
\text { temperature } \\
\text { distribution and } \\
\text { internal source }\end{array}$} & $\frac{d S_{\varphi f}(t)}{d t} \geq 0$ & $\int_{0}^{a}\left[\frac{\sum C_{l}\left(-\omega_{l}^{2}\right) e^{-\omega_{l}^{2} t}+\int_{0}^{t} D_{l}\left(-\omega_{l}^{2}\right) e^{-\omega_{l}^{2}(t-\tau)} d \tau+f(r, t)}{\sum C_{l} e^{-\omega_{l}^{2} t}+\int_{0}^{t} \sum D_{l} e^{-\omega_{l}^{2}(t-\tau)} d \tau}\right] r^{2} d r \geq 0$ \\
\hline & $S_{\varphi f}\left(t_{1}\right) \leq S_{\varphi f}\left(t_{2}\right)$ & $\begin{array}{l}\int_{0}^{a}\left[\ln \left(\sum_{l=0}^{\infty} A_{l} e^{-\omega_{l}^{2} t_{1}} j_{0}\left(k_{l} r\right)+\int_{0}^{t_{1}} \sum_{l=0}^{\infty} B_{l} e^{-\omega_{l}^{2}\left(t_{1}-\tau\right)} j_{0}\left(k_{l} r\right) d \tau\right)\right] r^{2} d r \\
\leq \int_{0}^{a}\left[\ln \left(\sum_{l=0}^{\infty} A_{l} e^{-\omega_{l}^{2} t_{2}} j_{0}\left(k_{l} r\right)+\int_{0}^{t_{2}} \sum_{l=0}^{\infty} B_{l} e^{-\omega_{l}^{2}\left(t_{2}-\tau\right)} j_{0}\left(k_{l} r\right) d \tau\right)\right] r^{2} d r\end{array}$ \\
\hline & $S_{\varphi f}(0) \leq S_{\varphi f}(\infty)$ & $\int_{0}^{a}[\ln \varphi(r)] r^{2} d r \leq \int_{0}^{a}\left[\ln \left(A_{0}+\lim _{t \rightarrow+\infty} \int_{0}^{t} B_{0} d \tau\right)\right] r^{2} d r$ \\
\hline
\end{tabular}

With the adiabatic boundary, the entropy generation is bounded by its values corresponding to that at volume average temperature of its initial temperature distribution [Eq. (9)] with the heat conduction driven by the initial temperature distribution, but unbounded [Eqs. (32) and (49)] if the heat conduction involves an internal source with a positive volume average over the sphere. As entropy reflects the part of the system energy that cannot be used to do useful work, it is critical to limit the use of nuclear, electrical, chemical, gammy-ray, or other sources that could produce positive average heat generation in adiabatic systems for the interest of our future.

\section{Conclusion}

By its very essence, entropy represents the part of system energy that cannot be transformed into useful work and is always generated in all processes. The present work of examining one-dimensional heat-conduction process in an adiabatic sphere shows a finite value of entropy generation as the time tends to infinity when the heat conduction is initiated by the initial temperature distribution, but infinity when the conduction involves positive heat source even in its average sense. Such an analysis is important by noting that our job is to make sure that entropy generation is well-controlled, our future depends also on whether the entropy generation is bounded. Therefore, we should constrain ourselves in using nuclear, electrical, chemical, gammy-ray, or other sources that could yield positive average heat generation in adiabatic systems.

By applying the second law of thermodynamics in analyzing heat conduction, we have also obtained mathematical inequalities of new or classical nature. This not only builds up the relation between mathematical inequalities and the second law of thermodynamics, but also offers an innovative way of studying differential equations. The obtained inequalities form a group of benchmarks relations useful for examining accuracy of analytical, numerical and experimental findings as well. 


\section{Acknowledgements}

The financial support from the Research Grants Council of Hong Kong (GRF 17237316, 17211115 and 17207914) and the University of Hong Kong (URC 201411159074 and 201311159187) is gratefully acknowledged. The work is also supported in part by the Zhejiang Provincial, Hangzhou Municipal and Lin'an County Governments.

\section{References}

[1] L. Q. Wang, X. S. Zhou and X. H. Wei, Heat Conduction: Mathematical Models and Analytical Solutions. SpringerVerlag, Heidelberg, 2008.

[2] X. W. Tian, X. Lai, P. A. Zhu and L. Q. Wang, "Beyond the classical theory of heat conduction: a perspective view of future from entropy," Proceedings of Royal Society A, vol. 472, p. 20160362, 2016.

[3] A. Bejan, Heat Transfer. John Wiley \& Sons, New York, 1993.

[4] L. Q. Wang, "Generalized Fourier law," International Journal of Heat and Mass Transfer, vol. 37, pp. 2627-2634, 1994.

[5] Y. A. Cengel and M. A. Boles, Thermodynamics: An Engineering Approach, 7th ed. McGraw-Hill, New York, 2011.

[6] A. Bejan, Advanced Engineering Thermodynamics, 4th ed, Wiley, Newark, 2016.

[7] L. Q. Wang, "Minimum heat to environment and entropy," International Journal of Heat and Mass Transfer, vol. 41, pp. 1869-1871, 1998.

[8] X. J. Zhu, X. Du, Y. Q. Ding and Q. G. Qiu, "Analysis of entropy generation behavior of supercritical water flow in a hexagon rod bundle," International Journal of Heat and Mass Transfer, vol. 114, pp. 20-30, 2017.

[9] D. L. Goodstein, Thermal Physics: Energy and Entropy. Cambridge University Press, Cambridge, 2015.

[10] A. Bejan, Entropy Generation Minimization: the Method of Thermodynamic Optimization of Finite-size Systems and Finite-time Processes. CRC Press, New York, 1996.

[11] S. Hussain, S. E. Ahmed and T. Akbar, "Entropy generation analysis in MHD mixed convection of hybrid nanofluid in an open cavity with a horizontal channel containing an adiabatic obstacle," International Journal of Heat and Mass Transfer, vol. 114, pp. 1054-1066, 2017.

[12] G. F. Naterer and J. A. Camberos, Entropy-based Design and Analysis of Fluids Engineering Systems. CRC Press, Boca Raton, 2008.

[13] T. Choudhary and S. Sanjay, "Thermodynamic assessment of SOFC-ICGT hybrid cycle: energy analysis and entropy generation minimization," Energy, vol. 134, pp. 1013-1028, 2017.

[14] I. Dinçer and M. A. Rosen, Exergy Analysis of Heating, Refrigerating and Air Conditioning: Methods and Applications. Elsevier, Amsterdam, 2015.

[15] M. G. Sobamowo and A. T. Akinshilo, "Analysis of flow, heat transfer and entropy generation in a pipe conveying fourth grade fluid with temperature dependent viscosities and internal heat generation," Journal of Molecular Liquids, vol. 241, pp. 188-198, 2017.

[16] J. E. Ahern, the Exergy Method of Energy Systems Analysis. Wiley, New York, 1980.

[17] C. E. Damian-Ascencio, A. Saldana-Robles, A. Hernandez-Guerrero and S. Cano-Andrade, "Numerical modeling of a proton exchange membrane fuel cell with tree-like flow field channels based on an entropy generation analysis," Energy, vol. 133, pp. 306-316, 2017.

[18] V. P. Singh, Entropy Theory in Hydrologic Science and Engineering. McGraw-Hill, New York, 2015.

[19] E. F. Beckenbach and R. Bellman, Inequalities. Springer-Verlag, Berlin, 1965.

[20] G. H. Hardy, J. E. Littlewood and G. P’olya, Inequalities. Cambridge University Press, Cambridge, 1934. 